\title{
Primary pulmonary malignant melanoma: a clinicopathologic study of two cases
}

\author{
Li Gong ${ }^{1}$, Xiao-Yan Liu', Wen-Dong Zhang', Shao-Jun Zhu', Li Yao', Xiu-Juan Han', Miao Lan', \\ Yan-Hong $\mathrm{Li}^{1,2,3^{*}}$ and Wei Zhang ${ }^{1,3^{*}}$
}

\begin{abstract}
Malignant melanoma involving the respiratory tract is nearly always metastatic in origin, and primary tumors are very rare. To our knowledge, about 30 cases have been reported in the English literature, one of which involved multiple brain metastases. Here, we report two cases of primary pulmonary malignant melanoma. The first case, which occurred in a 52-year-old Chinese female patient who died 4 months after the initial diagnosis, involved rapid intrapulmonary and intracranial metastases. The second patient, a 65-year-old female, underwent surgical excision, and clinical examination, histopathological characteristics, and immunohistochemical features supported the diagnosis of pulmonary malignant melanoma. No evidence for recurrence and/or metastasis has been found more than one year after the initial surgery. To establish the diagnosis of primary pulmonary malignant melanoma, any extrapulmonary origin must be excluded by detailed examination. Moreover, the tumor should be removed surgically whether it occurs as a single lesion or multiple lesions.
\end{abstract}

Virtual slide: The virtual slide(s) for this article can be found here: http://www.diagnosticpathology.diagnomx.eu/vs/ 1480477335765055.

Keywords: Primary pulmonary melanoma, Surgical resection, Chemotherapy, Metastasis, Treatment

\section{Introduction}

Malignant melanoma occurs most frequently on the skin, but can also arise in other organs and tissues of the body. However, primary pulmonary malignant melanoma is exceedingly rare [1]. To date, about 30 cases have been reported in the English literature [2], one of which involved multiple brain metastases [3]. This report presents two cases of primary malignant melanoma of the lung, and one case involves intrapulmonary and intracranial metastasis. The goal of this study was to illustrate the importance of establishing the diagnosis of primary pulmonary malignant melanoma by detailed examination upon diagnosis to exclude any extrapulmonary origin. Moreover, the tumor should be removed surgically whether it occurs as a single lesion or multiple lesions.

\footnotetext{
* Correspondence: lyhzhw@fmmu.edu.cn; zhwlyh@fmmu.edu.cn

${ }^{1}$ The Helmholtz Sino-German Laboratory for Cancer Research, Department of Pathology, Tangdu Hospital, The Fourth Military Medical University, Xi'an 710038, P.R China

${ }^{3}$ Department of Pathology, Tangdu Hospital, The Fourth Military Medical University, Xi'an 710038, P.R China

Full list of author information is available at the end of the article
}

\section{Case presentation \\ Case 1}

A 52-year-old Chinese female was admitted to our hospital because of a persistent cough. Previous computed tomography $(\mathrm{CT})$ of the chest revealed multiple illdefined masses in the upper and lower lobe of the left lung. Clinical examinations and routine laboratory tests were within normal limits. Comprehensive dermatologic and ophthalmologic examinations revealed no evidence of cutaneous or ocular primary melanoma. Bronchoscopic examination showed a bulging lesion in the bronchus of the lower left lobe, and a bronchial mucosal biopsy was performed. Histopathologically, the tumor cells were submucosal, and were composed of malignant epithelioid cells with large amounts of acidophilic cytoplasm and prominent nuclei. Mitotic figures were easily found. Dark brown pigment was observed in some tumor cells (Figure 1). Thus, our initial diagnosis was malignant melanoma. Immunohistochemical staining to confirm this diagnosis demonstrated that the tumor cells were positive for pan melanoma, S-100 protein, Vimentin, and HMB45 (melanoma marker) (Figure 2), and were negative for cytokeratin (CK), epithelial membrane 

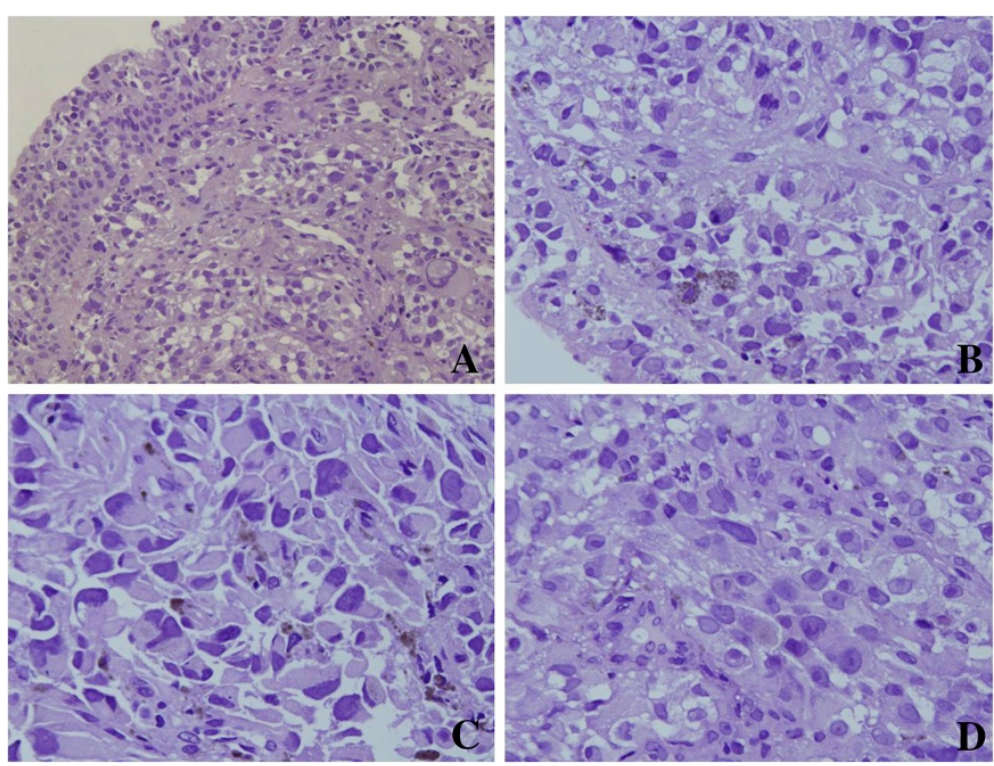

Figure 1 Histopathological characteristics of pulmonary malignant melanoma: The tumor cells located under bronchial mucosa, and was composed of malignant epithelioid tumor cells with large amounts of acidophilic cytoplasm and prominent nuclei. Mitotic figures were easily found. Dark brown pigment were observed in some tumor cells (A, ×200; B, C, and D, ×400).

antigen (EMA), chromaffin A (CgA), synaptophysin (Syn), high molecular weight cytokeratin, small cell lung cancer (SCLC), Desmin, smooth muscle actin (SM-actin), neuron specific enolase (NSE), leukocyte common antigen (LCA), and CD34. Based upon these histological characteristics, immunohistochemical features, and the fact that there was no evidence of malignant melanoma elsewhere, the final diagnosis was primary pulmonary malignant melanoma. The patient underwent regular adjuvant chemotherapy with dacarbazine. Three months after the initial diagnosis, the patient felt intermittent dizziness, and a cranial CT showed a solitary lesion in the occipital lobe. The physician thought that the intracranial lesion might be a metastatic focus, and advised
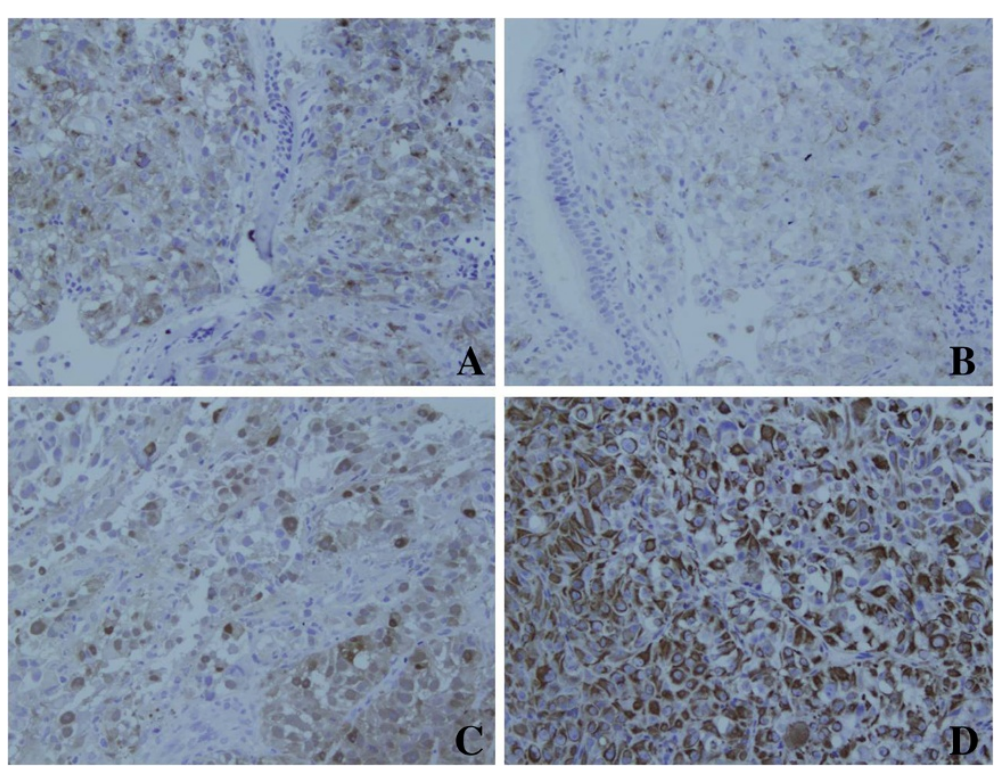

Figure 2 Immunohistochemical features of pulmonary malignant melanoma: The results of immunohistochemical staining demonstrated the tumor cells were positive for pan- Melanoma (Figure 2A, $\times 200$ ), HMB45 (Figure 2B, $\times 200$ ), S-100 protein (Figure 2C, ×200), and Vimentin (Figure 2D, ×200). 
the patient to begin therapy. However, she refused to undergo treatment and left hospital. At the end, she died 4 months after the initial diagnosis.

\section{Case 2}

A 65-year-old Chinese female was admitted to our hospital due to shortness of breath and weakness of 8 months' duration. She was initially treated with antibiotics for 1 week at home. However, her condition did not improve, and she was admitted to our hospital for further studies. A chest CT revealed a spherical mass in the lower lobe of the right lung. Her cranial CT was normal (Figure 3). Clinical examinations and routine laboratory tests were within normal limits. The patient demanded and received surgical treatment. Grossly, the mass measured $6 \times 4 \times 4 \mathrm{~cm}$, the cut surface was dark, and the texture was solid. Histological examination revealed the features of malignant melanoma with predominantly epitheliod cells and nuclei (Figure 4). This was further confirmed by immunohistochemistry, which showed that the tumor cells expressed pan melanoma, HMB45, and S-100 protein (Figure 4). As the same as the first patient, various examinations demonstrated that there was no lymph node metastasis or evidence of melanoma elsewhere. The final diagnosis was primary pulmonary malignant melanoma. Thus, the patient began adjuvant chemotherapy with dacarbazine three weeks after operation. To date, she is alive one year and six months after initial diagnosis. Moreover, there is no evidence of recurrence and/or metastasis.

\section{Discussion}

Worldwide, approximately 160,000 new cases of melanoma are diagnosed each year, and about 41,000 melanomarelated deaths occur annually [4]. Malignant melanoma mainly occurs on the skin, but has also been described in other mucosal sites and organs, including the oral cavity, paranasal sinuses, esophagus, larynx, vagina, anorectal region, and liver $[5,6]$. Primary malignant melanoma of the lung is an extremely rare non-epithelial neoplasm that accounts for only $0.01 \%$ of all primary lung tumors [7]. To date, about 30 cases have been reported in the English literature [2]. The mean age at diagnosis is 57 years (range 41-82). Although it appears to be dominant in males, both cases described in this report were female.

The precise histogenesis of pulmonary malignant melanoma remains controversial. Most experts believe that melanocytes migrate concomitantly with reduced growth of the primordial tubular respiratory tract during fetal growth [8]. Others think that these cases are a metastatic form of an antecedent skin lesion that is either unrecognized or has spontaneously regressed [9]. In addition, there are some likely explanations regarding the presence of melanoma in the lung, such as the possibility that melanocytes and melanocytic proliferations are present in the larynx and esophagus, or that the larynx, esophagus, and lungs all share a common embryologic origin, suggesting the possible migration of melanocytes [10].

Pathologically, primary pulmonary malignant melanoma resembles that of the skin or mucosa, and exhibits morphologic variability within the tumor sample. Microscopically, the tumor is composed of epithelioid cells arranged in nests, or spindle cells arranged in fascicles, with or without melanin pigment deposition. Mitotic figures are readily apparent. In both of our cases, the tumor cells were mainly submucosal and showed diffuse infiltration. They were pleomorphic, with round, spindle-shapes, irregular morphologies, and prominent nuclei. Some tumor cells contained melanin deposits. Thus, in similar cases, malignant melanoma should be the first consideration. However, immunohistochemical staining should also be performed to further confirm this diagnosis, and to exclude other melanotic tumors, such as melanotic medullary carcinoma of thyroid [11], and pigmented neuroendocrine carcinoma [12]. In our two cases, immunohistochemical staining demonstrated that the tumor cells expressed HMB45, S-100, pan melanoma and Vimentin, and did not express CK, EMA, CgA, Syn, HCG, HMW-CK, Desmin, SM-actin, TTF-1, and SCLC. Thus, the diagnosis was reliable, although there was no evidence of

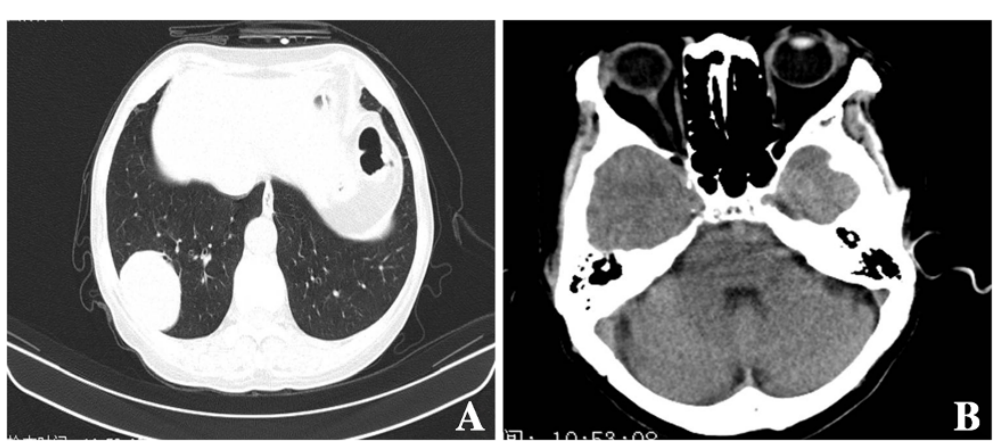

Figure 3 Chest and cranial CT of the second patient: A chest Computed tomography (CT) revealed a spherical mass in the right lung lower lobe (Figure $3 \mathrm{~A}$ ), and cranial CT showed normal (Figure $3 \mathrm{~B}$ ). 


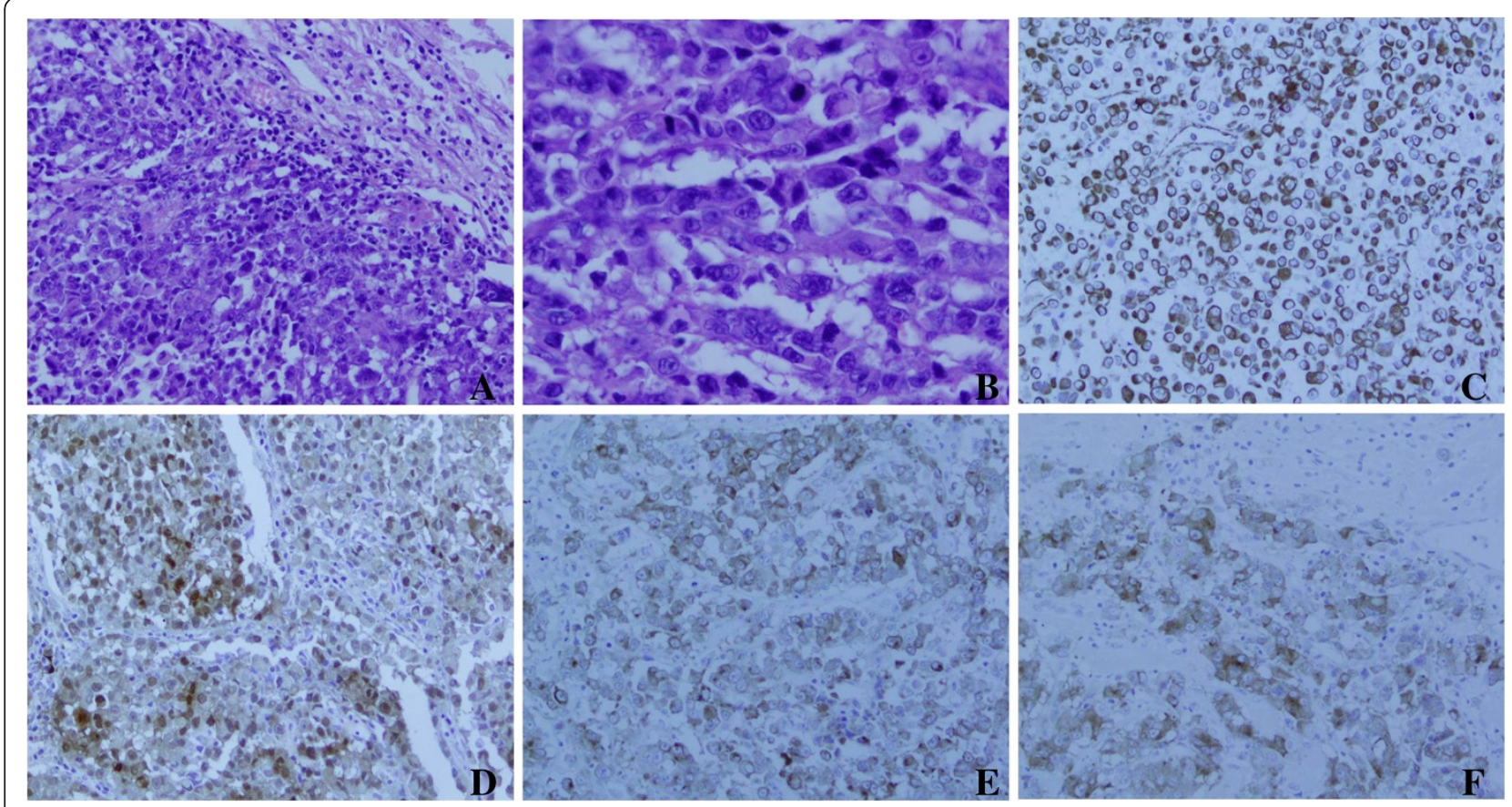

Figure 4 Histopathological characteristics and immunohistochemical features of the second case of pulmonary malignant melanoma: Histopathologically, the tumor was composed of malignant epitheliod tumor cells with large amounts of acidophilic cytoplasm and prominent nuclei. Mitotic figures were easily found. Lung tissue could be found in the surrounding of tumor cells (Figure 4A $\times 200$ and $4 \mathbf{B} \times 400$ ). Immunohistochemically, the tumor cells were positive for vimentin (Figure $4 \mathbf{C} \times 200$ ), S-100 protein (Figure 4D $\times 200$ ), melanoma-pan (Figure $4 \mathbf{E}$ $\times 200$ ), and HMB45 (Figure $4 \mathbf{F} \times 200$ ).

melanoma from transmission electron microscopic examination.

According to the published literature, approximately $5-10 \%$ of patients with metastatic melanoma have a primary melanoma of unknown origin $[13,14]$. Various reasons, such as occult cutaneous or visceral location, complete regression, or primary origin in lymph nodes due to malignant transformation of ectopic nevus cells, have been suggested $[15,16]$. Multiple nodules of the lung are generally considered intrapulmonary metastases. There were multiple lesions in our first case, so we had to determine whether the tumor was a primary or secondary lesion. To this end, we performed an extensive examination for the patient, including physical examinations, gastrointestinal endoscopy, colonoscopy, endoscopy of the nasalcavity, and positron emission tomographic scanning of the brain. The results showed no evidence of malignant melanoma elsewhere. In addition, CT did not reveal a solitary lesion in the occipital lobe upon initial admission. Moreover, according to the clinical and pathological criteria proposed by Allen and Drash and others $[1,8,17]-1)$ no history suggestive of a previous melanoma; 2) no demonstrable melanoma in any other organ at the time of surgery; 3) a solitary tumor in the surgical specimen from the lung; 4) tumor morphology compatible with that of a primary tumor; 5) no evidence at autopsy of a primary melanoma elsewhere; 6) obvious melanoma cells confirmed by immunohistochemical staining for S-100 and HMB-45, and possibly by electron microscopy; 7) evidence of junctional change; 8) "nesting" of cells beneath the bronchial epithelium; 9) invasion of the intact bronchial epithelium by melanoma cells - we concluded that the aforementioned data were indicative of primary lung melanoma with intrapulmonary metastasis. Moreover, we considered that the ninth view described in the above diagnostic criteria was an important characteristic of primary pulmonary melanoma besides the evidence of histopathological and immunohistochemical staining according to the features of our both cases. The tumor cells of primary pulmonary melanoma seemed also to involve in the bronchial epithelium.

The optimal treatment for patients with primary malignant melanoma of the lung remains to be determined. Some studies have demonstrated that the prognosis for surgically-resected patients is better than that for nonsurgically treated patients [2]. Our second case confirms this viewpoint. Of course, various chemotherapeutic agents, including dacarbazine and immunotherapy with interleukin-2 or interferon should be used as well. However, a cranial CT revealed a solitary lesion in the occipital lobe 3 months after the initial diagnosis for the first 
patient. We concluded that this was a probable metastatic malignant melanoma though a biopsy was not performed. Therefore, we feel that an aggressive surgical approach is warranted.

\section{Conclusion}

In conclusion, the extrapulmonary origin of malignant melanoma must be excluded by detailed examination to establish the diagnosis of primary pulmonary malignant melanoma. The tumor should be removed surgically whether it occurs as a single lesion or multiple lesions.

\section{Consent}

Written informed consent was obtained from the patients for publication of this report and any accompanying images.

\section{Abbreviations}

CgA, Chromaffin A; CK, Cytokeratin; CT, Computed tomography;

EMA, Epithelial membrane antigen; HMW-CK, High molecular weightcytokeratin; SCLC, Small cell lung cancer; LCA, Leukocyte common antigen; NSE, Neuron specific enolase; SM-actin, Smooth muscle actin;

Syn, Synaptophysin.

\section{Competing interests}

The authors declare that they have no competing interests.

\section{Authors' contributions}

GL conceived of the study and drafted the manuscript. LXY, YL, ZSJ, and HXJ participated in its acquisition of data and analysis. ZWD participated in drafting the manuscript. LM carried out the immunohistochemical assays. ZW and LYH participated in its design and coordination and helped to draft the manuscript. All authors read and approved the final manuscript.

\section{Acknowledgements}

This work was supported by The National Natural Science Foundation of China (No. 30800417) and The National Basic Research Program (973 Program ) of China ( No. 2009CB521705).

\section{Author details}

'The Helmholtz Sino-German Laboratory for Cancer Research, Department of Pathology, Tangdu Hospital, The Fourth Military Medical University, Xi'an 710038, P.R China. ${ }^{2}$ Department of Gynaecology and Obstetrics, Tangdu Hospital, the Fourth Military Medical University, Xi'an 710038, P.R China. ${ }^{3}$ Department of Pathology, Tangdu Hospital, The Fourth Military Medical University, Xi'an 710038, P.R China.

Received: 15 July 2012 Accepted: 15 September 2012

Published: 19 September 2012

\section{References}

1. Scolyer RA, Bishop JF, Thompson JF: Primary Melanoma of the lung. In Textbook of Uncommon Cancer. 3rd edition. Edited by Raghavan D, Brecher ML, Johnson DH, Meropol NJ, Moots PL, et al. West Sussex: John Wiley \& Sons Ltd; 2006:293-298.

2. Seitelman E, Donenfeld P, Kay K, Takabe K, Andaz S, Fox S: Successful treatment of primary pulmonary melanoma. J Thorac Dis 2011, 3(3):207-208.

3. Maeda R, Isowa N, Onuma H, Miura H, Tokuyasu H, Kawasaki Y: Primary malignant melanoma of the lung with rapid progression. Gen Thorac Cardiovasc Surg 2009, 57(12):671-674.

4. Parkin DM, Bray F, Ferlay J, Pisani P: Global cancer statistics, 2002. CA Cancer J Clin 2005, 55:74-108.

5. Ost D, Joseph C, Menezes G: Primary pulmonary melanoma: Case report and literature review. Mayo Clinic Proc 1999, 74:62-66.

6. Gong L, Li YH, Zhao JY, Wang XX, Zhu SJ, Zhang W: Primary malignant melanoma of the liver: a case report. World J Gastroenterol 2008, 14(31):4968-4971.
7. Dountsis A, Zisis C, Karagianni E, Dahabreh J: Primary malignant melanoma of the lung: A case report. World J Surg Oncol 2003, 1:26.

8. Jensen OA, Egedorf J: Primary malignant melanoma of the lung. Scand J Respir Dis 1967, 48:127-135.

9. Bagwell SP, Flynn SD, Cox PM, Davison JA: Primary malignant melanoma of the lung. Am Rev Respir Dis 1989, 139:1543-1547.

10. Jennings TA, Axiotis CA, Kress Y, Carter D: Primary malignant melanoma of the lower respiratory tract. Am J Clin Pathol. 1990, 94:649-655.

11. Singh K, Sharma MC, Jain D, Kumar R: Melanotic medullary carcinoma of thyroid - report of a rare case with brief review of literature. Diagnostic Pathology 2008, 3:2.

12. Schneider U, Ihle V, Kohler B, Meier D, Frenzel H: Pigmented neuroendocrine carcinoma of the hepatic duct: a case report. Diagnostic Pathology 2007, 2(Suppl 1):S11.

13. Serna MJ, Vazquez-Doval J, Sola MA, Ruiz de Erenchun F, Quintanilla E: Metastatic melanoma of unknown primary tumour. Cutis 1994, 53:305-308.

14. Chang AE, Karnell LH, Menck HR: The National Cancer Data Base report on cutaneous and noncutaneous melanoma: a summary of 84,836 cases from the past decade. The American College of Surgeons Commission on Cancer and the American Cancer Society. Cancer 1998, 83:1664-1678.

15. Katz KA, Jonasch E, Hodi FS, Soiffer R, Kwitkiwski K, Sober AJ, Haluska FG: Melanoma of unknown primary: experience at Massachusetts General Hospital and Dana-Farber Cancer Institute. Melanoma Research 2005, 15:77-82.

16. Baab GH, McBride CM: Malignant melanoma: the patient with an unknown site of primary origin. Arch Surg 1975, 110:896-900.

17. Allen MS Jr, Drash EC: Primary melanoma of the lung. Cancer 1968 21:154-9

doi:10.1186/1746-1596-7-123

Cite this article as: Gong et al:: Primary pulmonary malignant melanoma: a clinicopathologic study of two cases. Diagnostic Pathology 2012 7:123.

\section{Submit your next manuscript to BioMed Central and take full advantage of:}

- Convenient online submission

- Thorough peer review

- No space constraints or color figure charges

- Immediate publication on acceptance

- Inclusion in PubMed, CAS, Scopus and Google Scholar

- Research which is freely available for redistribution 